\title{
REALIZATION OF OFDM BASED UNDERWATER ACOUSTIC COMMUNICATION
}

\author{
Vijay A ${ }^{1}$, Sunil Jacob ${ }^{2}$, Shelma Jacob.K ${ }^{3}$ \\ ${ }^{1}$ Professor,Anvijay@Live.Com \\ ${ }^{2}$ Professor,Suniljacob01@Gmail.Com \\ ${ }^{3}$ M.Tech Scholar, Jacobshelma@Gmail.Com
}

\begin{abstract}
Nowadays underwater communication plays a vital role in applications from commercial extends to military purposes. Present underwater communication systems involve the transmission of information in the form of sound, electromagnetic (EM), or optical waves. All these techniques has their own benefits and limitations. Acoustic communication is the most versatile and widely used technique in underwater environments because of its low attenuation compared with others. Acoustic waves are more applicable for thermally stable, deep water settings. But acoustic waves in shallow water can be adversely affected by temperature gradients, surface ambient noise, and multipath propagation due to reflection and refraction. The much slower speed of acoustic propagation in water, about $1500 \mathrm{~m} / \mathrm{s}$ (meters per second), compared with that of electromagnetic and optical waves, and is another limiting factor for efficient communication and networking. Nevertheless, the currently favorable technology for underwater communication is upon acoustics.
\end{abstract}

In this paper, we are planning to design a simple underwater acoustic system. We first discuss about the problems of underwater communication. Then we are designing a data transmission system in underwater and its analysis is done in the next step.

Keywords: Underwater acoustic communication, Orthogonal frequency division multiplexing, Differential phase shift keying

\section{INTRODUCTION}

Underwater communication has a vital role in future technology.As we all know that our earth consists of $70 \%$ of water.Since wired communication has the practical limitations in under water,we have to develop wireless network that can work under water.It plays an important role in investigating climate change, heterogenous characteristics of seawater,oil production facilities and harbors using unmanned underwater vehicles(UUVs), submarines, and in underwater wireless sensor networks(UWSN),

As in all communication networks, there will be a medium for data transmission between transmitter and receiver. In underwater the possible ways of data transmission are EM waves, optical waves and acoustic waves.Even though EM waves have high speed, they can be used onlyup to a frequency range of less than $300 \mathrm{~Hz}$ because of their conducting nature in seawater.Also for using EM waves require high power transmitters and antennas[10]. Similarly most of the optical energy will get lost because of scattering while using optical waves for transmission. So the possible way of transmission is acoustic waves.

In order to improve the efficiency we are using a modulation by Orthogonal Frequency Division Multiplexing(OFDM) technique for acoustic waves. The results show satisfactory results. OFDM is motivated because of simple implementation and its capability to travel over long times read channels. In OFDM the frequency selective bandwidth is divided into narrower flat fading sub channels. OFDM transmits signals over these multiple orthogonal sub-carriers simultaneously and performs robustly in severe multi- path environments achieving high spectral efficiency and higher data transmission rate. Also while using OFDM the need of complex time-domain equalizers can be omitted, due to its robustness against frequency selective fading and narrowband interference.The first section contains the system model in which channel model is derived.The next section contains the analysis and results.

\section{SYSTEM MODEL}

Underwater acoustics communication is the communication between the underwater nodes by acoustic (sound) waves. Mainly it uses the frequency range of $10 \mathrm{~Hz}-1 \mathrm{MHz}$.But as frequency increases the loss rate also increases. A sound wave propagating underwater consists of alternating compressions and rarefactions of the water. These compressions and rarefactions are detected by a receiver, such as the human ear or a hydrophone, as changes in pressure.

We have to take care about the underwater characteristics before designing an UWA system. They are heterogeneous characteristics of underwater, attenuation due to absorption, multipath fading due to reflection and refraction, loss due to Doppler effect and noises in underwater. Because of the heterogeneous characteristics the speed of sound in water is about $1500 \mathrm{~m} / \mathrm{s}$.Soundspeed in water increases with 
increasing pressure, temperature and salinity. The maximum speed in pure water under atmospheric pressure is attained at about $74^{\circ} \mathrm{C}$; sound travels slower in hotter water after that point; the maximum increases with pressure. Though some sounds can travel remarkable distance through the water, higher frequency sound is absorbed much faster than low frequency. The main cause of sound absorption is the presence magnesium sulphate and boric acid, and geometrical spreading.

The frequency of the received sound will be different from that of the sound radiated because of the relative motion between transmitter and receiver. This change in frequency is known as a Doppler shift. The frequency drift due to Doppler Effect cause the overlapping of frequency channel,and thus, spread the channel further apart and occupying awider frequency range[10]. Because of this attenuation occurs at receiverside. The ambient noise in underwater is mainly due to four sources. They are turbulence noise, shipnoise, winddriven noise and thermal noise. These noises are frequency dependent and also depend on windspeed, shipping factor etc.Thermal noise increase with frequency whereas the remaining noiseseffect at lower frequencies.

\subsection{UNDERWATER CHANNEL MODEL}

The crucial part of underwater communication is the modeling of an underwater channel.The acoustic waves underthe water absorbed due to thespreading of the wave energy and energy density also decays[10]. Thus, the first model is to formulate the relationbetween the transmission loss with respect to the acousticchannel characteristic and the distance of transmission asthe attenuation model. Attenuation that take place in an underwater acousticcommunication over a distance $d[\mathrm{~m}]$ for a signal withfrequency $\mathrm{f}[\mathrm{kHz}]$ can be calculated using the Thorp model[1] which can be written as

$$
A(d, f)=d^{g} a(f)^{d}
$$

Where, g represents the spreading factor and a(f) $[\mathrm{dB} / \mathrm{km}]$ isthe absorption coefficient. Value $g$ indicates the geometry of propagation; typical values are $\mathrm{g}=2$ for spherical spreading, $\mathrm{g}=1$ for cylindrical spreading and $\mathrm{g}$ $=1.5$ for the alleged practical spreading. The absorption coefficient can be calculated by using the Throp's formula as

$$
\begin{gathered}
10 \log a(f)=0.11 \frac{f^{2}}{1+f^{2}}+44 \frac{f^{2}}{4100+f^{2}}+2.75 * 10^{-4} \\
* f^{2}+0.003
\end{gathered}
$$

As the absorption coefficientincreases rapidly with frequency, attenuation due to absorption at high frequencies causes large loss.

The four main sources of noise in an underwater system are: turbulence, shipping, waves, and thermal noise. These noises can be modeled by using Wenz model as the functions of frequency in $\mathrm{kHz}[3]$.

$$
\begin{gathered}
10 \log N_{t}(f)=17-30 \log (f) \\
10 \log N_{s}(\mathrm{f})=40+20(\mathrm{~s}-0.5)+26 \log (\mathrm{f})-60 \log (\mathrm{f}+0.003) \\
10 \log N_{w}(f)=50+7.5 w^{0.5}+20 \log (f)-40 \log (f) \\
+0.4) \\
10 \log N_{t h}(f)=-15+20 \log (f) \\
N(f)=N_{t}(f)+N_{s}(f)+N_{w}(\mathrm{f})+N_{t h}(f)
\end{gathered}
$$

$\mathrm{N}(\mathrm{f})$ represents the overall PSD of total noise in $\mathrm{dB}$.

The effect of noise on the signal is dependent on the signal to noise ratio (SNR), which can be formulated as.

$$
\operatorname{SNR}(d, f)=P /(A(d, f) N(f) \Delta f)
$$

Where dis distance, $f$ is the signal frequency and $\Delta$ Fis the receiver noise bandwidth. The value of $\mathrm{s}$ denotes the shipping activity factor which normally ranges from 0 to1.Similarly windspeed is denoted by w. Since underwater is a frequency selective fading channel, multipath in underwater can be modeled as Rayleigh fading whose coefficients are random.

\subsection{OFDM in underwater}

Single carrier transmission means one radio frequency carrier is used to carry the information. Hence information in the form of bits carried by one single RF carrier. In OFDM,also known as multicarrier modulation, uses multiple carriers sending some bits on each channel. InOFDM[8,17],all of the subchannels are dedicated to a data source.Multicarrier modulation especially OFDM is suitable for multipath environment as its carriers are orthogonal. As symbol period increases, the error rate is small compared with single carrier. Similarly the data rate is more for multicarrier modulation. But the problem of peak to average ratio (PAPR) arises in case of OFDM.

In this paper DPSK is used for subcarrier modulation for OFDM[18].In DPSK, input binary sequence is first differentially encoded and then modulated using a BPSK modulation[15].It reduces the receiver complexity as we do not need any channel estimation and save precious spectral efficiency.

\section{SIMULATION AND RESULTS}

Absorption can be modeled by using thorp model and the obtained figure $\mathrm{i}$ ass shown in figure 1 . 


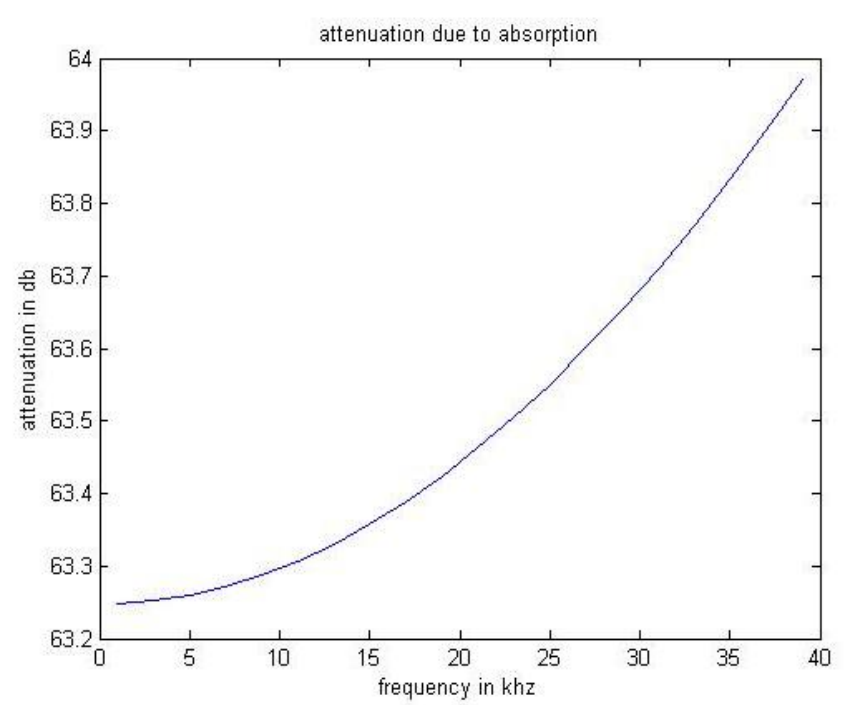

Figure 1

Since attenuation increases with frequency, wecan not take large frequencies. Using the Wenz model the attenuation due to noise is plotted for a frequency range of 1 to 40 $\mathrm{kHz}$. The shipping noise activities can be modeled through the shipping activity factor $\mathrm{s}$ where by the value is ranging between 0 and 1 for low and high activity, respectively.Figure2 shows shipping noise for different values of $\mathrm{s}$.

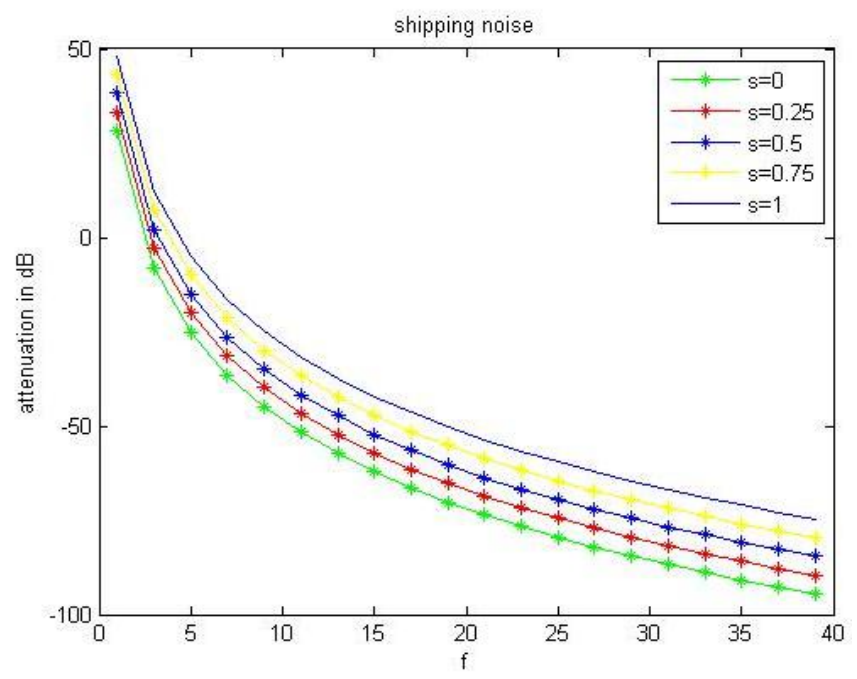

Figure2

In our project we are taking 0.5 for s. Surface motion, caused by winddrivenwaves depend on windspeedw, which is normally varying from 0 to $50 \mathrm{~m} / \mathrm{s}$. Figure shows the attenuation due to various values windspeed.

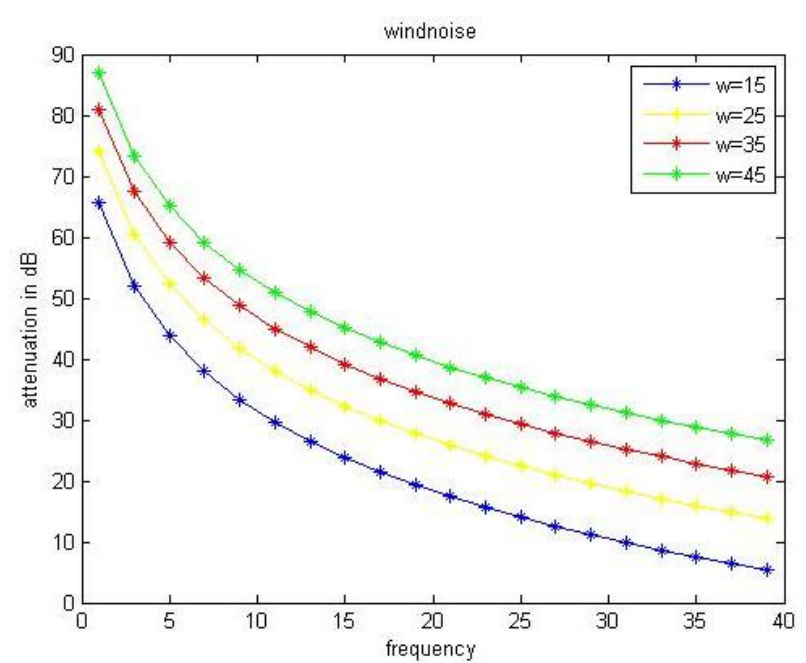

Figure3

Here for designing the project $\mathrm{w}$ is set to be 25

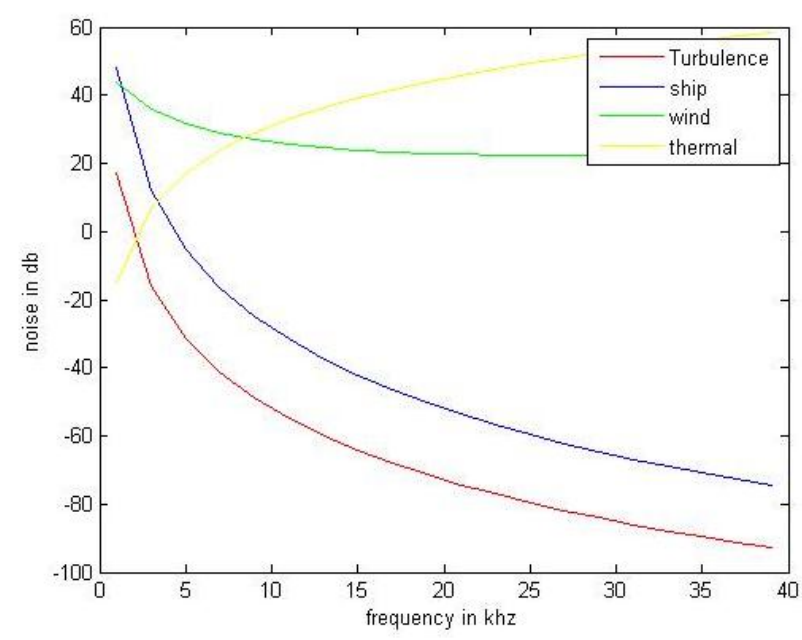

Figure4

From the figure4, it is understood that turbulence noise, shipping noise and wind noise are decreasing with frequency but thermal noise drastically increase with frequency. The frequency response of underwater channel obtained as

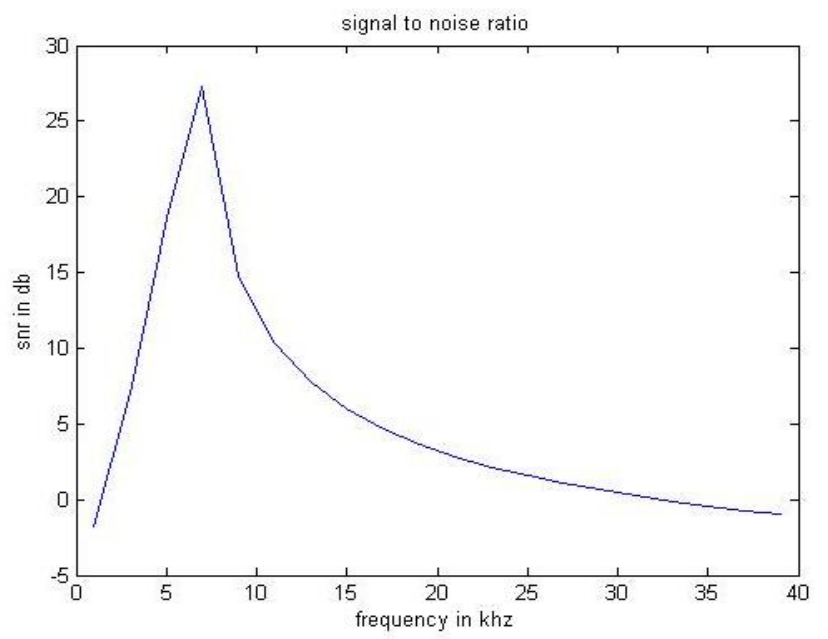

Figure5 
From the figure 5 it is clear that maximum snr obtains at a frequency range is 4 to $10 \mathrm{kHz}$. As transmitting power increases ,SNR value will increase.So here we are taking this frequency range for OFDM transmission between two fixed nodes.

The obtained bit error rate corresponding to the 64 bit and 128bitOFDM transmissionare tabulated in table1.

\begin{tabular}{|l|l|l|l|}
\hline $\begin{array}{l}\text { Modulation } \\
\text { Scheme }\end{array}$ & $\begin{array}{l}\text { No of bits } \\
\text { transmitted }\end{array}$ & $\begin{array}{l}\text { No of } \\
\text { errors }\end{array}$ & BER \\
\hline DPSK & 64 & 4 & 0.0625 \\
\hline 4-QAM & 64 & 12 & 0.1875 \\
\hline DPSK & 128 & 35 & 0.2734 \\
\hline 4-QAM & 128 & 52 & 0.4063 \\
\hline
\end{tabular}

Table1

It is clear that OFDM with DPSK modulation perform better than other modulation. But the error rate in underwater is very large compared with that of air.

\section{CONCLUSION}

This paper presented a study on modeling of underwater acoustic wireless communication with a detail model on the channelcharacteristic, environmental noise, signal to noise ratio.From this analysis it is understood that OFDM with DPSK modulation offers better performance while comparing with other schemes,since underwater is multipathn environment. Optimum frequencies are obtained at 4 to $12 \mathrm{KHz}$ for a small transmission distance with the highest SNR.

\section{ACKNOWLEDGEMENT}

I hereby acknowledge my deep sense of gratitude towards Prof. Vijay A, Prof. Saira Joseph and Dr. Sunil Jacob without whom this project would not have come into existence. We express our sincere thanks to Prof. R. Sahadevan (HOD of Electronics and Communication Department) and all other faculties who have guided us throughout this project.

\section{REFERENCES}

[1]. R. Coates, "Underwater Acoustic Systems," New York, Wiley, 1989

[2]. ]Bridget Benson, Ying Li, Brian Faunce, Kenneth Domond, Don Kimball, Curt Schurgers, and Ryan Kastner ,"Design of a Low-Cost Underwater Acoustic Modem "IEEE Journal of, vol. 21, pp. 6773,1998

[3]. E. M. Sozer, M. Stojanovic, and J. G. Proakis,"Underwater acoustic networks," Oceanic Engineering, IEEE Journal of, vol. 25, pp. 72 83,2000.

[4]. R. van Nee and R. Prasad, "OFDM for Wireless Multimedia Communications," Artech House, Inc., Norwood 2000

[5]. Mandar Chitre1, Shiraz Shahabudeen1, Lee Freitag2, Milica Stojanovic3," Recent Advances in Underwater Acoustic Communications \& Networking”, Acoustic
Research Laboratory, National University of Singapore, 2006

[6]. M. Stojanovic, "On the Relationship Between Capacity and Distancein an Underwater Acoustic Communication Channel," WUWNet'06,Los Angeles, California, USA, 2006.

[7]. Lanbo Liu1, Shengli Zhou2 and Jun-Hong Cui3,"Prospects and problems of wireless communication for underwater sensor networks", Wirel. Commun. Mob. Comput. 2008; 8:977-994

[8]. Sung-Jun Hwang and Philip Schniter, "Efficient Multicarrier Communication for Highly Spread Underwater Acoustic Channels" ,IEEE Journal On Selected Areas In Communications, VOL. 26, NO. 9, DECEMBER 2008

[9]. Shalomi Arnon ,"Underwater optical communication" ,Optical Engineering 491, 015001 January 2010

[10]. Hou Pin Yoong, KiamBeng Yeo, Kenneth Tze Kin Teo and Wei Loong Wong,"Underwater Wireless Communication System: Acoustic Channel Modeling andCarry Frequency Identification",ISSN: 11 473804x online

[11]. P.Vijaya Kumar, S.S.K.Praneeth, Romarsha.B.Narender, "Analysis of Optical Wireless Communication for Underwater Wireless Communication", International Journal of Scientific \& Engineering Research Volume 2, Issue 6, June20111 ISSN 2229-5518.

[12]. C. Polprasert, J. A. Ritcey and M. Stojanovic, "Capacity of OFDM Systems over Fading Underwater Acoustic Channels," IEEE Journal of Oceanic Engineering, Vol. 36, No. 4, 2011, pp. 514524.

[13]. C. R. Berger, J. Gomes and J. M. Moura, "Study of Pilot Designs for Cyclic-Prefix OFDM on TimeVarying and Sparse Underwater Acoustic Channels," OCEANS, San- tander, 6-9 June 2011, pp. 1-8.

[14]. J. Heidemann, M. Stojanovic, and M. Zorzi, "Underwater sensor networks: Applications, advances, and challenges", Phil. Trans. R. Soc. A, pp. 158-175, Jan. 2012.

[15]. Z. Wang, S. Zhou, G. B. Giannakis, C. R. Berger and J. Huang, "Frequency-Domain Oversampling for Zero-Pad- ded OFDM in Underwater Acoustic Communications," IEEE Journal of Oceanic Engineering, Vol. 37, No. 1,2012 Chengsheng Pan, Liangchen Jia, Ruiyan Caiand Yuanming Ding," Modeling And Simulation Of Channel For Underwater'International Journal of Innovative Computing, Information and Control ,Volume 8, Number 3(B), March 2012

[16]. Hamada Esmaiel, Danchi Jiang ," Review Article: Multicarrier Communication for Underwater Acoustic Channel" Int. J.Communications, Network and System Sciences, 2013, 6, 361-376

[17]. Arnaud Bourr'e, Said Lmai, Christophe Laot and S'ebastien Houcke ," A Robust OFDM Modem for Underwater Acoustic Communications", IEEE 2013. 


\section{BIOGRAPHY}

Shelma Jacob. $\mathbf{K}$ received her B.Tech degree from Sahrdaya College of Engineering and Technology,University of Calicut in 2012.She is currently pursuing her M.Tech degree in Communication Engineering from SCMS School of Engineering and Technology,Karukutty.

Email:jacobshelma@gmail.com 